\title{
Expert camouflage-breakers can accurately localize search targets
}

\author{
Fallon Branch (1), Allison JoAnna Lewis, Isabella Noel Santana and Jay Hegdé* (1)
}

\begin{abstract}
Camouflage-breaking is a special case of visual search where an object of interest, or target, can be hard to distinguish from the background even when in plain view. We have previously shown that naive, non-professional subjects can be trained using a deep learning paradigm to accurately perform a camouflage-breaking task in which they report whether or not a given camouflage scene contains a target. But it remains unclear whether such expert subjects can actually detect the target in this task, or just vaguely sense that the two classes of images are somehow different, without being able to find the target per se. Here, we show that when subjects break camouflage, they can also localize the camouflaged target accurately, even though they had received no specific training in localizing the target. The localization was significantly accurate when the subjects viewed the scene as briefly as $50 \mathrm{~ms}$, but more so when the subjects were able to freely view the scenes. The accuracy and precision of target localization by expert subjects in the camouflage-breaking task were statistically indistinguishable from the accuracy and precision of target localization by naive subjects during a conventional visual search where the target 'pops out', i.e., is readily visible to the untrained eye. Together, these results indicate that when expert camouflage-breakers detect a camouflaged target, they can also localize it accurately.
\end{abstract}

Keywords: Accuracy, Categorization, Pop-out, Precision, Visual search

\section{Significance}

In order to recognize a foreground visual object of interest, or target, camouflaged against its background, the viewer must be able to perceptually segregate it from the rest of the image. By definition, effectively camouflaged objects are hard to detect.

We have previously demonstrated the somewhat counterintuitive fact that ordinary, naive subjects can learn to accurately recognize the camouflaged target by learning the statistical properties of the background (Chen \& Hegdé, 2012a). Briefly, subjects were trained using a deep learning task in which they were shown individual camouflage scenes and were required to report whether or not the image contained a target object. Subjects were

\footnotetext{
*Correspondence: jhegde@augusta.edu

Department of Neuroscience and Regenerative Medicine, Medical

College of Georgia, Augusta University, CA-2003, 1469 Laney Walker Blvd,

Augusta, GA 30912-2697, USA
}

provided feedback after their response, which served to implicitly and retroactively label the image as one in which the target was present or absent. Importantly, subjects were not told what to learn or what the target object was, nor shown the target in isolation (Chen \& Hegdé, 2012b; Streeb et al., 2012). Over several hundred trials (depending on the subject), the subjects' performance improved to highly significant levels. This occurred regardless of the target object and even when the subjects were never shown the same image twice, so that the only way the subjects could learn the task was by learning the statistical properties of the background, i.e., what a given background 'looked like', so that they could tell when the image contained an 'odd-man-out' object that did not share the statistics of the background.

Collectively, these observations suggest that, in order to successfully perform this task, the trained subjects distinguish the overall statistical properties of the images that do not contain a target from the properties of the images that 
do contain a target. But they also raise an important followup question: When such trained experts perform this task, do they detect the actual target, or simply sense that the two classes of images are somehow different? This issue is highly significant in real-world situations. For instance, our finding that naive subjects, regardless of whether or not they have any a priori aptitude for visual pattern recognition, can be trained to detect camouflaged targets even upon a very brief viewing (Chen \& Hegdé, 2012a); also see (Chen \& Hegdé, 2012b; Streeb et al., 2012) is potentially applicable to real-world combat situations. However, merely being able to judge, no matter how accurately, that the given combat scene contains a target is not very useful to a sniper under real-world combat conditions if he/she is unable to also accurately tell where the target is.

The localization issue is also significant from a more purely scientific point of view, especially given the fact that expert subjects perform our camouflage-breaking task accurately even when there is no physical target to localize, i.e., when the scene did not contain a physical target object, but simply had the overall statistical properties of images that did [see Fig. 4 of (Chen \& Hegdé, 2012a); also see (Chen \& Hegdé, 2012b; Streeb et al., 2012)]. Similar findings have been reported in breast cancer screening, where expert radiologists can accurately distinguish mammograms with versus without a lesion even when there is no visible lesion to localize (Brennan et al., 2018; Evans et al., 2016). Thus, it would seem that, at least in principle, localization is dissociable from detection; the latter can occur without the former. But it is unclear whether the ability to localize the target has to be learned separately, or whether it develops as a matter of course when subjects acquire the underlying pattern recognition expertise. Besides, there is evidence that the localization performance is not all that dissociable from detection performance in expert radiologists (Carrigan et al., , 2018, 2019).

We therefore sought to empirically measure the localization performance in expert subjects who had been trained in the camouflage-breaking task, but had received no training whatsoever in localization per se. We show both that when expert camouflage-breakers detect a camouflaged target, they can also localize it quite accurately (Experiment 1). Moreover, this localization performance is statistically indistinguishable from the target localization performance of naive observers in a classical pop-out visual search task (Experiment 2).

\section{Experiment 1: Localization of camouflaged targets by expert subjects \\ Methods \\ Subjects}

All procedures used in this study were reviewed and approved in advance by the Institutional Review Board
(IRB) of Augusta University in Augusta, GA, where this study was carried out. All subjects were adult volunteers who had normal or corrected-to-normal vision and provided written informed consent prior to participating in the study. All consenting subjects were enrolled in the study; no other inclusion or exclusion criteria were used.

Six subjects participated in Experiment 1. Prior to their participation in this experiment, subjects were trained to criterion using our previously described deep learning method to break camouflage (Chen \& Hegdé, 2012a). It is important to emphasize that what the subjects were trained in was a detection task, i.e., one in which they had to report whether or not a given image contained an unspecified camouflaged target (i.e., an object that 'did not belong'). The subjects received no specific training in, nor information, instructions, or feedback about, localizing the target. All of the subjects had reached an asymptotic camouflage-breaking performance of $d^{\prime} \geq 1.95$ $(p<0.05)$ during this 'offline' training prior to their participation in Experiment 1.

During Experiment 1, each subject performed 4 to 6 blocks (depending on the subject) of 40 trials each. Prior to the actual data collection, the task paradigm was explained to the subjects with the help of figures that pictorially illustrated each step of a typical trial, as well as the organization of the trial blocks and testing sessions. We gave the subjects ample opportunity to ask questions and verbally ascertained that they understood the task. Subjects performed practice trials [mean $4.17 \pm 0.75$ (SD)] to thoroughly familiarize themselves with the task paradigm before the actual experiment began. The data from the practice trials were discarded.

\section{Stimuli}

The stimuli used in this experiment were generated as previously described in detail (Chen \& Hegdé, 2012a). Briefly, we digitally synthesized a large number of camouflage scenes using (depending on the image) one of three types of naturalistic background textures ["fruit" (see Fig. 1), "foliage," or "nuts" (see, e.g., Figs. 1 and 4 of (Chen \& Hegdé, 2012a))].

A given scene had a $50 \%$ chance of containing a single target and $50 \%$ chance of containing no target at all. When the stimulus did contain a target, the target had $50 \%$ chance each of being a human head (see, e.g., Fig. 1a, left) or a novel, naturalistic 3D object called a "digital embryo" [see, e.g., Fig. 1a, right; also see (Chen \& Hegdé, 2012a; Hauffen et al., 2012]. To determine the pixel location of the target, we divided the image into an imaginary $8 \times 6$ grid, so that each cell of the grid was $2^{\circ} \times 2^{\circ}$. The target was centered in a random location within a randomly selected cell of the grid. We also varied the size of the target at three different scales, so that the longest 
a
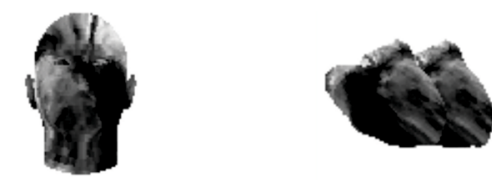

b

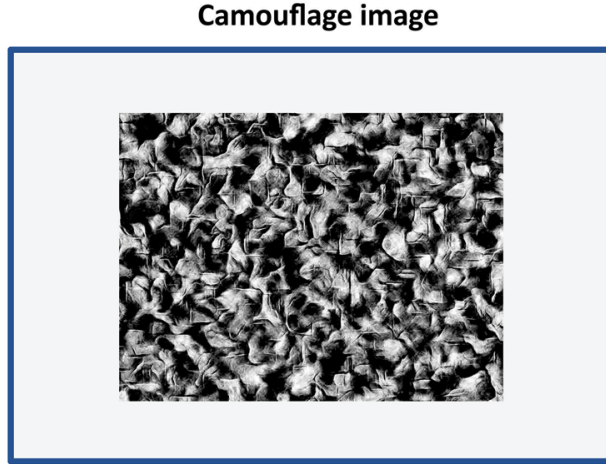

Stimulus viewing for $\mathbf{5 0} \mathrm{ms}$ or free viewing (depending on the trial)

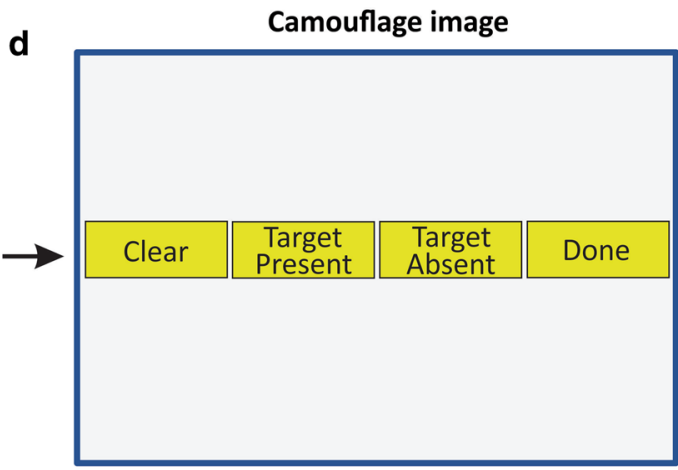

Duration: $60 \mathrm{~s}$
C

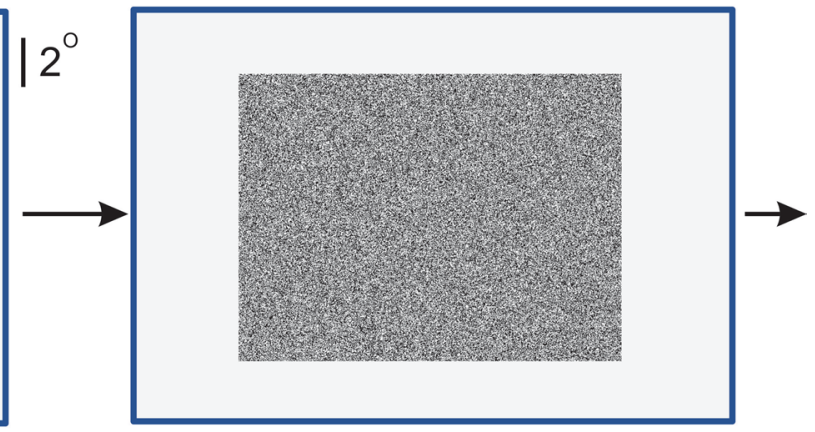

RDS mask: $500 \mathrm{~ms}$

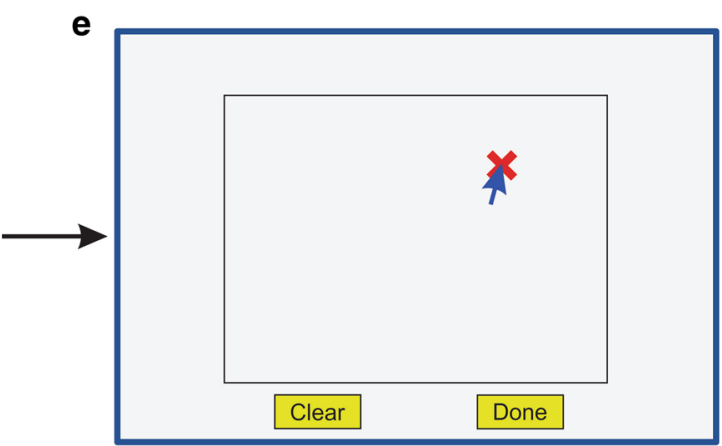

Duration: $60 \mathrm{~s}$

Fig. 1 Experiment 1: Visual search for camouflaged target. a Target objects. When a target was present, it was either a human head (left) or a digital embryo (right), each shown here at $4 \times$ their size in actual camouflage images. The human head target can be seen at the top right corner of the camouflage image in panel B. b-e Task paradigm. Subjects viewed the camouflaged scene (panel B), followed by a $0.5 \mathrm{~s}$ random dot stimulus mask (RDS mask; panel C). Subjects reported whether or not the preceding stimulus contained a target using designated onscreen buttons (panel D). Subjects were next presented with a blank outline of the image and were required to report the perceived location of the target (if the target was present) or the center of the image (if the target was absent) using a mouse click. An ' $X$ ' appeared at the clicked location (panel E). Not drawn to exact scale. See text for details

orthonormal aspect of the target was $\sim 0.1^{\circ}, 0.75^{\circ}$, or $0.5^{\circ}$, depending randomly on the image. Moreover, the target was rotated randomly about its $y$-axis from $-90^{\circ}$ to $+90^{\circ}$ depending on the image [see (Chen \& Hegdé, 2012a) for additional technical details].

The images used in this experiment belonged to the same texture and target class as that in which the given subject was trained. However, no image used during the training was re-used in this experiment, so that the images used during the training versus this experiment constituted two different, non-overlapping subsets of images randomly drawn from the same superset of stimuli.

\section{Procedure}

Each trial began when the subject fixated on a central fixation spot and indicated readiness by pressing a key on the computer's keyboard. A single $16^{\circ} \times 12^{\circ}$ camouflage scene was then presented as shown in Fig. $1 \mathrm{~b}$ in one of the following two conditions randomly interleaved in equal proportions within each trial block: (1) free viewing, or (2) time-limited (50 ms) viewing. In either 
condition, the subjects were able to end the stimulus presentation and proceed to the next phase of the trial by pressing a key. Depending on the trial, the stimulus location was randomly jittered by up to $1^{\circ}$. The camouflage scene was followed by a random dot stimulus (RDS) mask presented for $0.5 \mathrm{~s}$ (Fig. 1c).

As its name indicates, the RDS consisted of a random field of pixels, each of which had a $50 \%$ probability of being black or white.

After the mask was turned off, the subjects reported whether or not the preceding stimulus contained a target using on-screen buttons (Fig. 1, middle) and then used a mouse-click to report the perceived center of the target (if present) or the perceived center of the image (if the target was absent). To help determine if the localization performance was affected by whether the subjects had already reported their decision as to the presence or absence of the target, we swapped the order of the two reporting stages of the trial (denoted by panels $\mathrm{D}$ and $\mathrm{E}$ in Fig. 1) in two blocks each for three subjects (not shown). The data from these trial blocks were indistinguishable from the data with the original trial configuration (data not shown). The two sets of data were therefore pooled.

Data were analyzed using scripts custom-written for $R$ (r-project.org) or Matlab (Mathworks.com) platforms. Statistical tests for the accuracy of target localization were carried out using Hotelling's $T^{2}$ test in $R$. Correction for multiple comparisons was carried out using the false discovery rate (FDR) method (Benjamini \& Hochberg, 1995).

\section{Results and discussion}

Expert subjects can accurately localize camouflaged targets

\section{Detection performance}

As expected, the subjects were able to accurately detect the camouflaged target during the free-viewing condition [mean $d^{\prime}=2.39 \pm 0.29(\mathrm{SD}) ; p<0.05$ overall and for each subject]. The average reaction time was $598 \mathrm{~ms} \pm 96$. As also expected, during the $50 \mathrm{~ms}$ viewing, the detection performance was slightly lower, albeit still highly significant (mean $d^{\prime}=2.22 \pm 0.41 ; p<0.05$ overall and for each subject). The difference in the detection performance between the two conditions was statistically insignificant (Wilcoxon signed rank test; $V=7, p>0.05$ ).

The reaction times during this condition were slightly lower (589 ms \pm 103 ), although statistically indistinguishable from those during the free-viewing condition (Welch Two Sample $t$ test; $t=-1.55, d f=1162.2, p>0.05)$. This result also held when the reaction times were re-analyzed to take target status (i.e., present/absent) into account using a two-way ANOVA (condition $\mathrm{x}$ target status; $p>0.05$ for both factors and their interaction; also see
Table 1, top row). Together, the above results indicate that the differences in the detection accuracy between the two conditions were not attributable to a speed-accuracy tradeoff (Luce, 1991).

\section{Localization performance}

To determine whether the reported locations differed significantly from the actual location, we determined the reported locations across all subjects and trials. Since the actual location of the target varied randomly from one image to the next (see "Methods"), a principled method of comparing the localizations across all trials is to measure the subjects' reported localization during each given trial as the deviation from the actual physical location of the target during that trial.

The top left panel of Fig. 2 shows the results for freely viewed stimuli with targets. The degree to which the reported location deviates, or is separated, from the actual location of the target (denoted by the crosshair) constitutes the accuracy of localization (Dodge, 2003; Green \& Swets, 1966; Van Trees, 2001), so that a perfectly localized target would be denoted by a plotting symbol centered on the crosshair. The spread (or, in statistical terms, variance) of the reported locations from the actual location constitutes its precision (Dodge, 2003; Green \& Swets, 1966; Van Trees, 2001).

To determine whether the reported locations differed significantly from the actual location, we used a Hotelling's $T^{2}$ test, which, like many statistical tests of significance, balances the two-dimensional [2D] separation (i.e., accuracy) versus spread (i.e., precision) (Henkel, 1976; Hotelling, 1931). We used it to test the alternative hypothesis that the reported $2 \mathrm{D}$ target locations deviated significantly from the actual target's 2D location $(\mu=\{0,0\}$, denoted by the crosshair). We found that when the subjects were able to view the camouflage scene freely (Fig. 2, top left), the reported locations of the targets (red

Table 1 Measures of the precision of the localizations in Experiment 1

\begin{tabular}{|c|c|c|c|c|}
\hline & \multicolumn{2}{|c|}{ Target present } & \multicolumn{2}{|l|}{ Target absent } \\
\hline & Free viewing & $\begin{array}{l}\text { Stimulus } \\
\text { duration: } \\
50 \mathrm{~ms}\end{array}$ & Free viewing & $\begin{array}{l}\text { Stimulus } \\
\text { duration: } \\
50 \mathrm{~ms}\end{array}$ \\
\hline $\begin{array}{c}\text { Mean reac- } \\
\text { tion time } \\
(\mathrm{ms}) \pm S D\end{array}$ & $595 \pm 95$ & $586 \pm 103$ & $604 \pm 97$ & $593 \pm 103$ \\
\hline $\begin{array}{c}\text { Mean locali- } \\
\text { zation dis- } \\
\text { tance } \pm S D^{a}\end{array}$ & $1.36 \pm 0.68$ & $1.44 \pm 0.71$ & $1.38 \pm 0.73$ & $1.45 \pm 0.74$ \\
\hline
\end{tabular}

a Distance was calculated as the Euclidean distance (in degrees of arc) between the reported location of the target and its actual location during each trial 


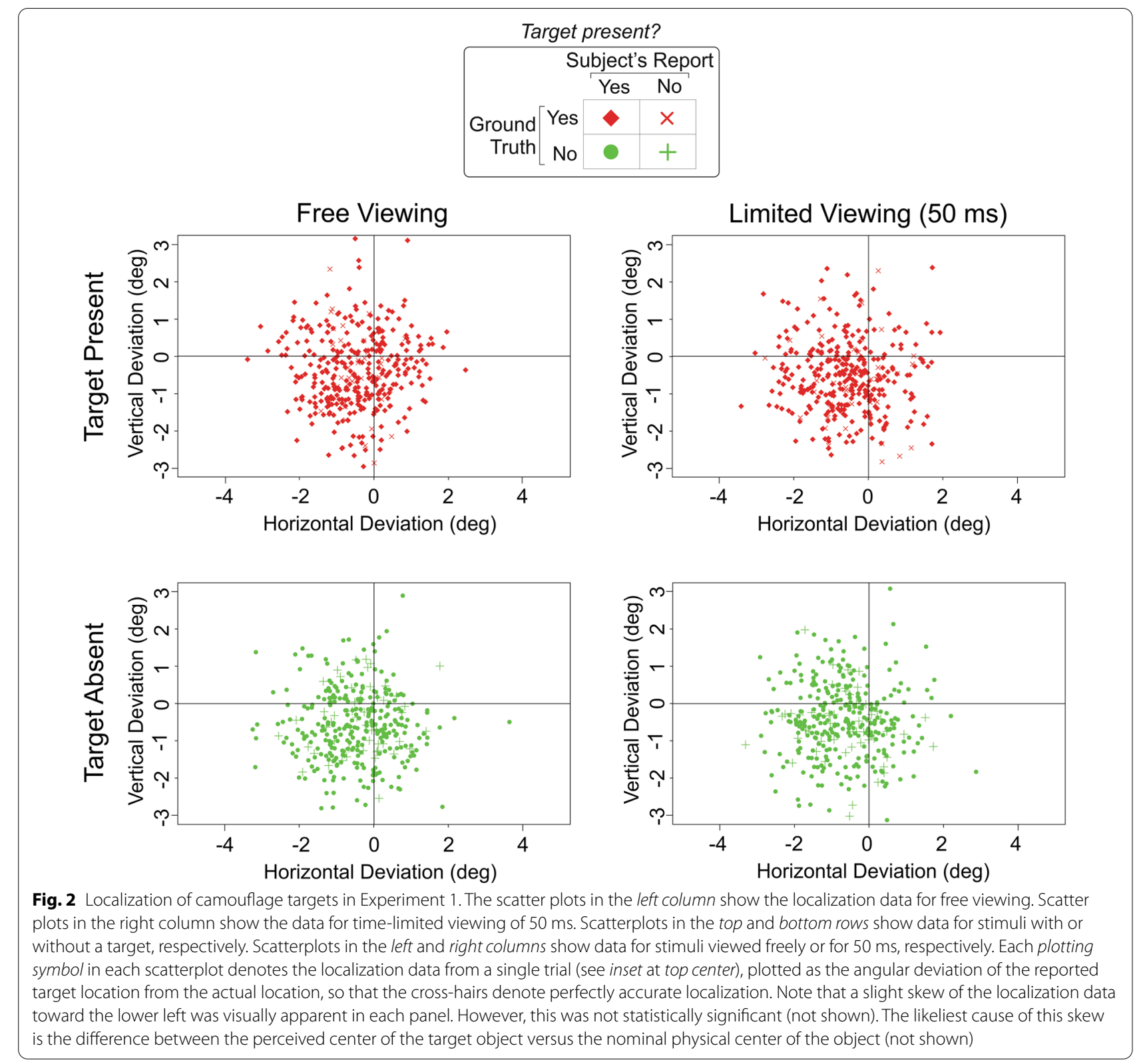

symbols) were statistically indistinguishable from the actual location of the target (center of crosshair; Hotelling's $T^{2}$ test, $F(1,700)=0.76, p>0.05$, FDR-corrected for multiple comparisons). Thus, when the subjects had ample opportunity to view the camouflage image, their reported target locations were non-random and were centered on the actual location of the target instead.

To help determine whether and to what extent the stimulus duration per se affected the accuracy of target localization, we examined the accuracy of localization for the stimulus duration of $50 \mathrm{~ms}$. Note that a stimulus duration this brief allows for little in the way of eye movements (Ibbotson \& Krekelberg, 2011; Kowler, 2011) [also see (Hess et al., 2016)]. Nonetheless, subjects were able to localize the target in this case as well (Fig. 2, top right; Hotelling's $T^{2}$ test, $F(1,700)=0.07, p>0.05$, FDRcorrected). Indeed, target localization after $50 \mathrm{~ms}$ viewing was statistically indistinguishable from localization after free viewing (Hotelling's $T^{2}$ test, $F(1,1398)=6.41$, $p>0.05$, corrected). That is, the subjects were able to localize the targets accurately even upon a brief viewing of the stimulus.

As noted in the Methods section, when the stimulus did not contain a target, subjects were instructed to report the location as the center of the stimulus. This provided a baseline measure for how accurate the subjects were 
in localizing the target. We found that the localization in these cases was statistically indistinguishable from localization when the target was present (Hotelling's $T^{2}$ tests, $p>0.05$, corrected).

Together, the above results show that subjects were, in a statistical manner of speaking, highly accurate in their localizations. But the localizations were self-evidently imprecise; the reported target locations deviated substantially from the actual target locations (see Fig. 2). Summary statistics shown in Table 1 (bottom row) indicate that the subjects misestimated the target location by about $1.45^{\circ}$ on average. The precision of target localization did not vary as a function of stimulus duration and of whether the stimulus contained a target (two-way ANOVA, stimulus duration $\times$ target status, $p>0.05$ for both factors and their interaction). This straightforwardly indicates that these factors were not the main source of the localization errors (see General Discussion below).

\section{Experiment 2. Localization of pop-out targets by naive subjects}

In this experiment, the stimuli consisted of conventional visual search arrays that are known to elicit perceptual 'pop-out' (Treisman, 1988; Wolfe, 1994). The rationale for using pop-out search arrays was that expert camouflage-breakers report that the subjective experience of searching for a camouflaged target is similar to searching for a pop-out target in a conventional visual search array (Chen \& Hegdé, 2012b), in that the target is effortlessly visible.

This experiment tested the hypothesis that the localization performance observed in Experiment 1 was not idiosyncratic to the camouflage-breaking task nor unusually imprecise, but was comparable to the target localization performance in other visual search tasks. In this experiment, subjects performed the same task as in Experiment 1 , but used visual search arrays where the target was readily recognizable (or 'popped-out'; see Methods for details; also see Fig. 3).

\section{Methods \\ Subjects}

Subject were recruited and consented exactly as in Experiment 1, except that seven subjects who did not participate in Experiment 1 participated in this experiment.

\section{Stimuli}

In this experiment, instead of camouflage scenes, conventional pop-out visual search arrays were used as stimuli. Since Experiment 1 featured six different types of stimuli ( 2 targets $\times 3$ background types), we used six different types of pop-out stimuli in this experiment: yellow $45^{\circ}$ bar target (when present) among blue $135^{\circ}$ bar distractors (Fig. 3a), black T-shaped target among white L-shaped distractors (Fig. 3b), yellow vertical bar target among yellow horizontal bar distractors (not shown), white Q-shaped target among white O-shaped distractors (not shown), black O-shaped target among black C-shaped distractors (not shown), and blue S-shaped target among green $\mathrm{H}$-shaped distractors(not shown). Each of these stimuli has been previously shown to result in perceptual pop-out ((Treisman, 1998); also see (Treisman, 1988; Wolfe, 1994)).

To help make the target location in this experiment analogous to the target location in Experiment 1, the arrays were created by dividing the stimulus into the aforementioned $8 \times 6$ grid, so that in the stimuli without a target, there were 48 distractors, one in each cell of the grid. The location of each distractor within its cell was randomly jittered by up to $0.35^{\circ}$ in a random direction. In the remaining stimuli, there was a single target that 'popped out', or was readily recognizable, located in a randomly selected cell of the grid, accompanied by 47 distractors in the remaining cells.
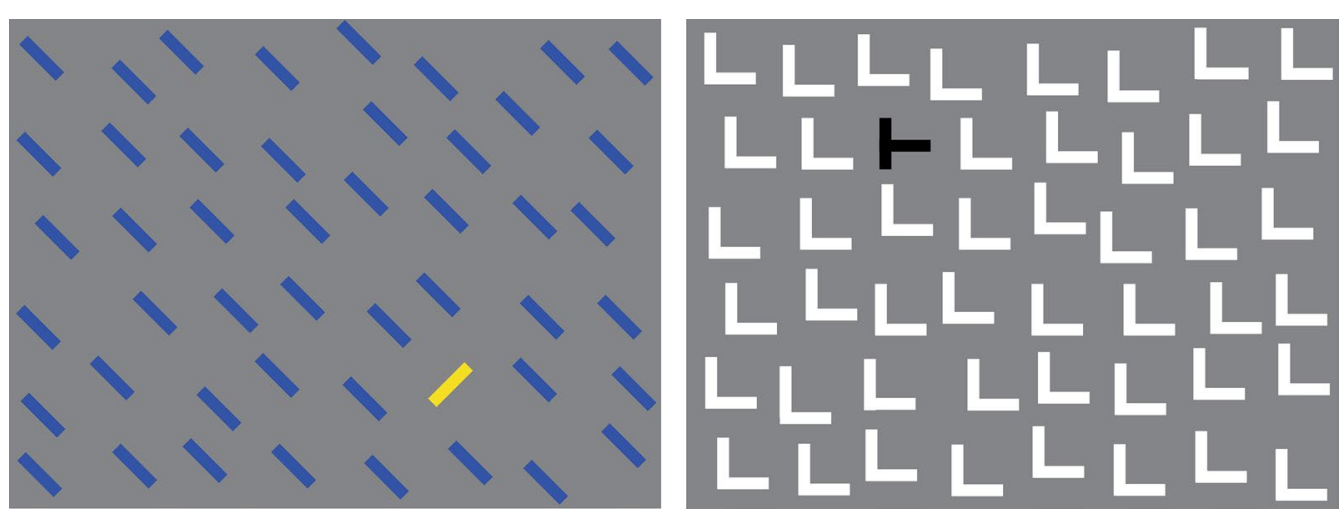

Fig. 3 Exemplar conventional visual search stimuli used in Experiment 2. See text for details 


\section{Procedure}

The procedure was identical to that used in Experiment 1 , except in the following respects: In this experiment, the aforementioned pop-out stimuli were used instead of camouflage images. Before the actual experiment, subjects carried out an average of $5.29 \pm 1.80$ practice trials. Subjects received no other training or practice of any kind.

\section{Results and discussion Detection performance}

The detection performance in this experiment was statistically indistinguishable from that in Experiment 1 using a three-way ANOVA (experiment $\times$ condition $\times$ target status; $p>0.05$ for all factors). Specifically, the subjects were able to accurately detect the pop-out target during the free-viewing condition [mean $d^{\prime}=2.27 \pm 0.29$ (SD); $p<0.05$ overall and for each subject]. The average reaction time was $634 \mathrm{~ms} \pm 95$. As also expected, during the $50 \mathrm{~ms}$ viewing, the detection performance was slightly lower, albeit still highly significant (mean $d^{\prime}=2.11 \pm 0.35$; $p<0.05$ overall and for each subject). The difference in the detection performance between the two conditions was statistically insignificant (Wilcoxon signed rank test; $V=5, p>0.05)$.

The reaction times during this condition ( $529 \mathrm{~ms} \pm 95)$ were statistically indistinguishable from those during the free-viewing condition (Welch Two Sample $t$ test; $t=-$ $0.96, d f=1241.1, p>0.05)$. This result also held when the reaction times were re-analyzed to take target status (i.e., present/absent) into account using a two-way ANOVA (condition $\times$ target status; $p>0.05$ for both factors and their interaction; also see Table 2, top row). Together, the above results indicate that the differences in the detection accuracy between the two conditions were not attributable to a speed-accuracy tradeoff in this experiment either.

Localization performance during the pop-out visual search is comparable to the localization performance in camouflage-breaking tasks.

We found that the results from this experiment were qualitatively similar to the results from Experiment 1 (Fig. 4 and Table 2). To quantitatively compare the results between the two experiments, we carried out a three-way analysis of variance (ANOVA) using three factors with two levels each: Experiment (1 vs. 2$) \times$ condition (freeviewing vs. $50 \mathrm{~ms}$ ) $\times$ target status (present vs. absent). We found that none of the factors or their interactions had a statistically significant effect on localization performance ( $p>0.05$ in each case). This result confirms the hypothesis that the localization performance Experiment 1 was not idiosyncratic to the camouflage-breaking task nor unusually imprecise, but was comparable to the
Table 2 Measures of the precision of the localizations in Experiment 2

\begin{tabular}{|c|c|c|c|c|}
\hline & \multicolumn{2}{|l|}{ Target present } & \multicolumn{2}{|l|}{ Target absent } \\
\hline & Free viewing & $\begin{array}{l}\text { Stimulus } \\
\text { duration: } \\
50 \text { ms }\end{array}$ & Free viewing & $\begin{array}{l}\text { Stimulus } \\
\text { duration: } \\
50 \mathrm{~ms}\end{array}$ \\
\hline $\begin{array}{l}\text { Mean reac- } \\
\text { tion time } \\
(\mathrm{ms}) \pm S D\end{array}$ & $637 \pm 95$ & $631 \pm 100$ & $627 \pm 96$ & $626 \pm 95$ \\
\hline $\begin{array}{c}\text { Mean locali- } \\
\text { zation dis- } \\
\text { tance } \pm S D^{a}\end{array}$ & $1.38 \pm 0.70$ & $1.52 \pm 0.78$ & $1.43 \pm 0.70$ & $1.45 \pm 0.73$ \\
\hline
\end{tabular}

a Distance was calculated as the Euclidean distance (in degrees of arc) between the reported location of the target and its actual location during each trial

target localization performance in the conventional parallel visual search (pop-out) tested in Experiment 2.

\section{General discussion}

Our results show that expert subjects can accurately tell where the camouflaged target is in a camouflage scene, and they can do so even upon viewing the stimulus as briefly as $50 \mathrm{~ms}$. This localization performance was statistically indistinguishable from the performance of naive subjects in a conventional pop-out search task. That is, trained camouflage-breakers can find a target in a camouflage scene as quickly and accurately as untrained subjects can find a target that pops-out of a conventional visual search array.

If the subjects were performing the task based on the perceived differences in the overall statistical properties of images with versus without a target, they would be able to localize the center of the image when the target was absent, because one can, in principle, do this by visually gauging the center of the image outline provided during the localization phase of the trial (Fig. 1, right panel). However, they would not be able to accurately localize the actual target using this strategy. Therefore, in this scenario, the localization performance can be expected to be better when the target was absent versus localization when the target was present. However, this was not what we empirically observed; the localization was statistically indistinguishable between the two cases, but accurate in both cases. Together, these considerations suggest that the subjects actually perceived the physical target when it was present. This was also consistent with the subjective percepts verbally reported by the subjects after their participation in the study was completed (not shown).

These findings have important potential applicability to real-world combat situations because they imply, taken together with the fact that the expert subjects were not trained specifically in the localization task, that 


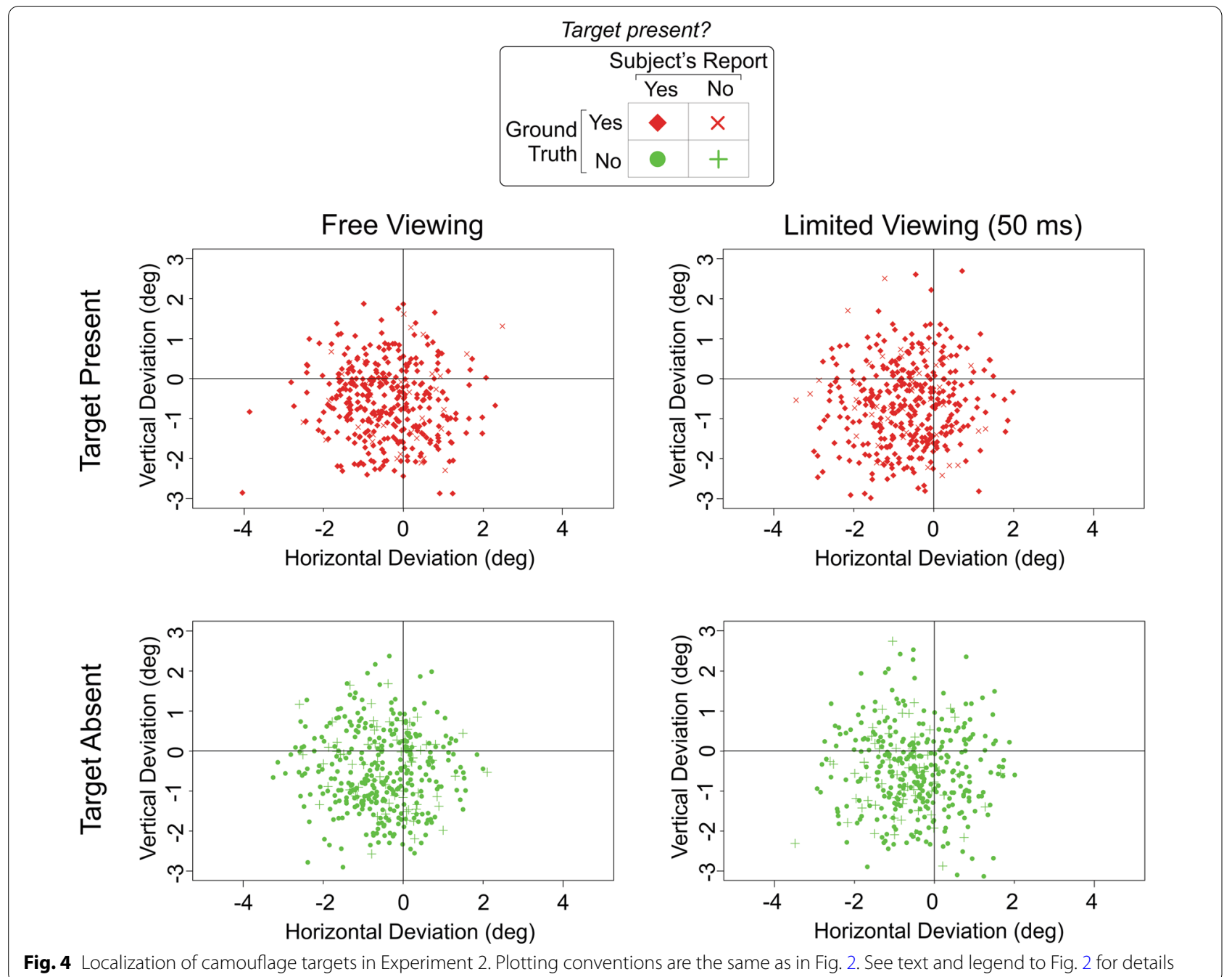

expert camouflage-breakers do not have to be specifically trained in the localization task. The localization expertise evidently develops as a matter of course of acquiring the target detection expertise using our deep learning paradigm. This raises additional intriguing questions as to whether this is idiosyncratic to our training paradigm, and whether and to what extent localization versus detection expertise develop concurrently during the training. Additional studies are needed to resolve these issues.

As noted above, while the expert camouflage-breakers were able to localize the camouflaged target as accurately as naive subjects localized targets that popped out, neither localization performance was not all that precise. After all, in real-world combat situations, a sniper who localizes the target with a precision of about $1.45^{\circ}$ of arc leaves much to be desired. It is possible that the poor precision is somehow an inherent limitation of our deep learning training protocol. However, this is unlikely to be the sole cause of the imprecision, especially in view of the fact naive subjects who received no training performed just as imprecisely using a substantially different type of stimuli. It is possible the errors are attributable, at least in part, to random and/or systematic motor errors [see, e.g., (Pelisson \& Prablanc, 2009)] and the fact that the target themselves varied in size from $0.5^{\circ}$ to $1^{\circ}$, depending on the trial. It is also worth noting that in both experiments, the stimulus had been turned off and masked by the time subjects got an opportunity to localize it. That is, our subjects localized a remembered target, and not a visible one. Previous studies using other sensorimotor tasks have shown that the neural information about target location decays, and the magnitude of localization errors increases, rapidly over time, especially in the absence of a visible localization target [see, e.g., (Binder et al., 2009; Pelisson \& Prablanc, 2009)]. Thus, it is plausible that similar temporal decay of the location information about 
the target contributed, in part, to the localization errors. A related issue is whether the precision of localization is different in higher-level categorization tasks, e.g., when the subjects have to determine if the human head is that of a friend or foe, compared to the low-level detection (target present or not?) task used in the present study. These and the aforementioned issues raised by the present preliminary study point to some useful future directions of research.

\section{Acknowledgements}

We thank Dr. Alan Saul for helpful discussions, and a Guest Editor and anonymous reviewers for useful suggestions. This study was supported by grant \# W91 1NF-15-1-0311 from the Army Research Office (ARO) to JH. AJL and INS were supported by the Undergraduate Research Apprentice Program (URAP) of the Army Educational Opportunity Program (AEOP) of the US Army.

\section{Authors' contributions}

All authors participated in designing the study. FB, AJL, and INS collected the data. FB and $\mathrm{JH}$ analyzed the data. All authors participated in preparing the manuscript and have approved the final version.

\section{Funding}

This study was supported by grant \# W911NF-15-1-0311 from the Army Research Office (ARO) to JH. AJL and INS were supported by the Undergraduate Research Apprentice Program (URAP) of the Army Educational Opportunity Program (AEOP) of the US Army.

\section{Availability of data and materials}

The images used in the study and the deidentified data are available for noncommercial use upon reasonable request.

\section{Declarations}

\section{Ethics approval and consent to participate}

All procedures used in this study were reviewed and approved in advance by the Institutional Review Board (IRB) of Augusta University in Augusta, GA, where this study was carried out. All subjects were adult volunteers and provided written informed consent prior to participating in the study.

\section{Consent for publication}

Not applicable.

\section{Competing interests}

None.

Received: 1 September 2020 Accepted: 17 March 2021

Published online: 06 April 2021

\section{References}

Benjamini, Y., \& Hochberg, Y. (1995). Controlling the false discovery rate: a practical and powerful approach to multiple testing. Journal of the Royal Statistical Society B, 57, 289-300.

Binder, M. D., Hirokawa, N., \& Windhorst, U. (2009). Motor Error. In M. D. Binder, N. Hirokawa, \& U. Windhorst (Eds.), Encyclopedia of neuroscience. (pp. 2437-2437). Springer.

Brennan, P. C., Gandomkar, Z., Ekpo, E. U., Tapia, K., Trieu, P. D., Lewis, S. J., Wolfe, J. M., \& Evans, K. K. (2018). Radiologists can detect the "gist" of breast cancer before any overt signs of cancer appear. Scientific Reports, 8(1), 8717. https://doi.org/10.1038/s41598-018-26100-5.

Carrigan, A. J., Curby, K. M., Moerel, D., \& Rich, A. N. (2019). Exploring the effect of context and expertise on attention: is attention shifted by information in medical images? Attention, Perception, \& Psychophysics, 81(5), 1283-1296. https://doi.org/10.3758/s13414-019-01695-7.

Carrigan, A. J., Wardle, S. G., \& Rich, A. N. (2018). Finding cancer in mammograms: if you know it's there, do you know where? Cogn Res Princ Implic, 3(1), 10. https://doi.org/10.1186/s41235-018-0096-5.

Chen, X., \& Hegdé, J. (2012a). Learning to break camouflage by learning the background. Psychological Science, 23(11), 1395-1403. https://doi.org/10. 1177/0956797612445315.

Chen, X., \& Hegdé, J. (2012b). Neural mechanisms of camouflage-breaking: A human fMRI study. Journal of Vision, 12, 582.

Dodge, Y. (Ed.). (2003). The oxford dictionary of statistical terms. (6th ed.). Oxford University Press.

Evans, K. K., Haygood, T. M., Cooper, J., Culpan, A. M., \& Wolfe, J. M. (2016). A half-second glimpse often lets radiologists identify breast cancer cases even when viewing the mammogram of the opposite breast. Proceedings of the National academy of Sciences of the United States of America, 113(37), 10292-10297. https://doi.org/10.1073/pnas.1606187113.

Green, D. M., \& Swets, J. A. (1966). Signal detection theory and psychophysics. . Wiley.

Hauffen, K., Bart, E., Brady, M., Kersten, D., \& Hegde, J. (2012). Creating objects and object categories for studying perception and perceptual learning. Journal of Visualized Experiments. https://doi.org/10.3791/3358.

Henkel, R. E. (1976). Tests of significance. . Sage Publications.

Hess, A. S., Wismer, A. J., Bohil, C. J., \& Neider, M. B. (2016). On the hunt: searching for poorly defined camouflaged targets. PLOS ONE, 11(3), e0152502. https://doi.org/10.1371/journal.pone.0152502.

Hotelling, H. (1931). The generalization of Student's ratio. Annals of Mathematical Statistics, 2(3), 360378. https://doi.org/10.1214/aoms/1177732979.

Ibbotson, M., \& Krekelberg, B. (2011). Visual perception and saccadic eye movements. Current Opinion in Neurobiology, 21(4), 553-558. https://doi.org/10. 1016/j.conb.2011.05.012.

Kowler, E. (2011). Eye movements: the past 25 years. Vision Research, 51(13), 1457-1483. https://doi.org/10.1016/j.visres.2010.12.014.

Luce, R. D. (1991). Response times: Their role in inferring elementary mental organization. . Oxford University Press.

Pelisson, D., \& Prablanc, C. (2009). Eye-Hand Coordination - Planning and Neural Structures. In M. D. Binder, N. Hirokawa, \& U. Windhorst (Eds.), Encyclopedia of neuroscience. (pp. 1540-1542). Springer.

Streeb, N., Chen, X., \& Hegdé, J. (2012). Learning-dependent changes in brain responses while learning to break camouflage: A human fMRI study. Journal of Vision, 12, 1131.

Treisman, A. (1988). Features and objects: the fourteenth Bartlett memorial lecture. Quarterly Journal of Experimental Psychology. A, Human Experimental Psychology, 40(2), 201-237. https://doi.org/10.1080/02724988843000104.

Treisman, A. (1998). Feature binding, attention and object perception. Philosophical Transactions of the Royal Society of London. Series B, Biological sciences, 353(1373), 1295-1306. https://doi.org/10.1098/rstb.1998.0284.

Van Trees, H. L. (2001). Detection, estimation, and modulation theory. . Wiley.

Wolfe, J. M. (1994). Guided Search 2.0 A revised model of visual search. Psychonomic Bulletin \& Review, 1(2), 202-238. https://doi.org/10.3758/BF032 00774.

\section{Publisher's Note}

Springer Nature remains neutral with regard to jurisdictional claims in published maps and institutional affiliations. 